Isomerie von Benzochinonoximen

\title{
II ${ }^{1}$ Stereoisomerie und Tautomerie von 1.4-Benzochinonoximen
}

\author{
Helmut UfFmann \\ Institut für Organische Chemie der Technischen Hochschule Hannover \\ (Z. Naturforschg. 22 b, 491—502 [1967] ; eingegangen am 30. Dezember 1966)
}

\begin{abstract}
Stereoisomere 1.4-Benzochinonoxime liegen in Lösung im Gleichgewicht vor; die Umwandlung erfolgt über ein mesomeres Ion.

Bei 1.4-Benzochinon-4-oximen, die in 3-Stellung substituiert sind, steht die Oximgruppe nur anti zu diesem Substituenten. Das sogenannte „stabile anti *-2-Chlor-5-methyl-1.4-benzochinon-4-oxim“ (, $\beta$-Form“) ist ein Addukt dieses Oxims (Oximgruppe syn zu Cl) mit dem strukturisomeren syn *. 2-Methyl-5-chlor-1.4-benzochinon-4-oxim.

Die Stereoisomeren von Chinonoximäthern lassen sich trennen; sie erleiden in Lösung bis $100{ }^{\circ} \mathrm{C}$ keine Umwandlung in das andere Stereoisomere.
\end{abstract}

1.4-Benzochinonoxim und 4-Nitrosophenol stehen im tautomeren Gleichgewicht miteinander. Im festen Zustand liegen die bisher untersuchten Verbindungen als Chinonoxime vor, worauf die Ähnlichkeit der IR-Spektren mit denen der Benzochinone ${ }^{2}$ und die vorliegenden Ergebnisse der Röntgen-Diffraktionsanalyse ${ }^{3}$ hinweisen. Umstrittener ist bis heute die Frage nach der Natur und der Lage des Tautomerengleichgewichts ${ }^{1,4}$.

Sehr wenig bekannt ist darüber, ob stereoisomere Chinonoxime existieren oder nicht. In der älteren Literatur - Zusammenfassung in l. c. ${ }^{5}$ - sind verschiedene Formen von acylierten oder alkoxylierten Chinonoximen beschrieben, die Stereoisomere sein sollen, aber auch vom 2-Chlor(Brom) -5-methyl-1.4benzochinon-4-oxim selbst liegen zwei Formen vor ${ }^{6}$. Als „einziges freies Chinon-oxim mit zwei stabilen Raumisomeren" hat es fortan eine wichtige Rolle gespielt. Mit chemischen Methoden war eine Aufklärung bisher nicht möglich, da Chinonoxime keine B e ckmann sche Umlagerung geben ${ }^{7}$. So ist die Stereoisomerie mehrfach angezweifelt worden. P. RAMart-Lucas z. B. ${ }^{8}$ hielt die " $\beta$-Form“ des 2 -Chlor1.4-benzochinon-4-oximethyläthers für ein verunreinigtes Präparat der „ $\alpha$-Form“, die entsprechenden

* Hier und im folgenden bezieht sich die Kennzeichnung „syn“ oder „anti“ immer auf den zuerst genannten Substituenten.

1 1. Mitt.: H. Uffmans, Tetrahedron Letters [London] 1966, 4631.

2 G. E. Philbrook u. T. G. Getten, J. org. Chemistry 24, 568 [1958].

3 C. Romers u. Mitarb., Rec. Trav. chim. Pays-Bas 76, 490 [1957].

4 A. Fischer, R. M. Golding u. W. C. Tennant, J. chem. Soc. [London] 1965, 6032.
Acetyl-Derivate des Oxims teils für den Nitrosophenolester, teils für das Oximacetat. In letzter Zeit hat sich vor allem eine Forschergruppe um Havinga und Romers mit diesem Problem befaßt ${ }^{9}$. Romers hat durch Röntgendiffraktions-Analyse die Raumisomerie der beiden 2-Chlor-1.4-benzochinon-4-oximacetate exakt bewiesen ${ }^{9}$. Von diesen Forschern wurden auch besonders eingehend die,$\alpha “$ - und die " $\beta$ “. Form des 2-Chlor(Brom) -5-methyl-1.4-benzochinon4-oxims untersucht, worauf später noch eingegangen werden muß.

\section{A. Orientierende Untersuchung der Protonenresonanz-Spektren}

Eigene Untersuchungen gingen davon aus, daß sich im 1.4-Benzochinonoxim aus der starren $\mathrm{C}=\mathrm{N}$ Bindung und dem $\mathrm{N}-\mathrm{O}$-Winkel eine unterschiedliche magnetische Abschirmung für das syn- und das anti-Proton in Nachbarstellung zur Oximgruppe wie bei Ketoximen ${ }^{10}$ und damit ein $\mathrm{ABXX}^{\prime}$ oder ein AMXX'-Spektrum für die Ringprotonen ergeben sollte. Vom 4-Nitrosophenol erwartet man dagegen wegen der freien Drehbarkeit der $\mathrm{N}=0$-Gruppe Äquivalenz der Protonen in 3.5-Position und damit

5 J. Meisenheimer u. W. Theilacker, in: K. Freudenberg, Stereochemie, Leipzig u. Wien 1933, S. 1082-1086.

${ }^{6}$ F. Kehrmann u. Mitarb., Liebigs Ann. Chem. 303, 1 [1898].

7 R. Raphael u. E. Vogel, J. chem. Soc. [London] 1952, 1958.

8 P. Ramart-Lucas u. M. Martynoff, Bull. Soc. chim. France 16, 59 u. 901 [1949].

9 Letzte Mitteilung über 1.4-Benzochinonoxime, C. Romers Mitarb., Acta crystallogr. [Copenhagen] 14, 753 u. 759 [1961].

10 E. Lustig, J. physic. Chem. 65, 491 [1961]. 


\begin{tabular}{|c|c|c|c|c|c|c|}
\hline Ringprotonen in Position von & 3 & & 5 & 2 und 6 & $J_{35}$ & $J_{23}=J_{56}$ \\
\hline 2-Methoxycarbonyl-4-nitrosophenol & 8,80 & & 7,65 & 7,06 & 2,4 & 9,0 \\
\hline 4-Nitrosoanisol & & 7,90 & & 7,16 & 2,5 & 9,2 \\
\hline 4-Nitrosophenol & & 7,85 & & 6,91 & & 9,0 \\
\hline 1.4-Benzochinon-4-oxim & 7,75 & & 7,22 & 6,43 & 2,5 & 10,3 \\
\hline Benzochinon-4-oximmethyläther & 7,64 & & 7,19 & 6,43 & 2,5 & 10,4 \\
\hline 2.6-Dimethyl-1.4-benzochinon-4-oxim & 7,53 & & 6,98 & & 2,5 & \\
\hline 2.6-Di-tert.butyl-benzochinon-4-oxim & 7,50 & & 6,90 & & 2,5 & \\
\hline
\end{tabular}

Tab. 1. Chemische Verschiebung $\delta$ (PPM) und Kopplungskonstanten $J$ (Hz) von Protonenresonanz-Spektren (4-Nitrosoanisol in Aceton, die anderen Verbindungen in Dioxan gelöst).

ein $\mathrm{AA}^{\prime} \mathrm{XX}^{\prime}$-Spektrum. Das Spektrum des Benzochinon-4-oximmethyläthers ist wie erwartet vom AMXX'-Typ mit einer chemischen Verschiebung von 0,45 PPM zwischen den Protonen in 3- bzw. 5-Stellung (Tab. 1). Vom Benzochinon-4-oxim erhält man jedoch in Deuteriumoxid, Dimethylsulfoxid, Aceton und Tetrahydrofuran nur $\mathrm{AA}^{\prime} \mathrm{XX}^{\prime}$-Spektren mit der Chem. Verschiebung $\delta^{*} \sim 7,6$ PPM und $\sim 6,6$ PPM. In einer ersten Mitteilung ${ }^{1}$ konnte gezeigt werden, daß dieses Verhalten nicht auf überwiegendes Vorliegen des Nitrosophenols, sondern auf die Besonderheit des Tautomerengleichgewichts mit nicht vernachlässigbarer Beteiligung des Resonanzhybridions ${ }^{11}$ zurückzuführen ist:

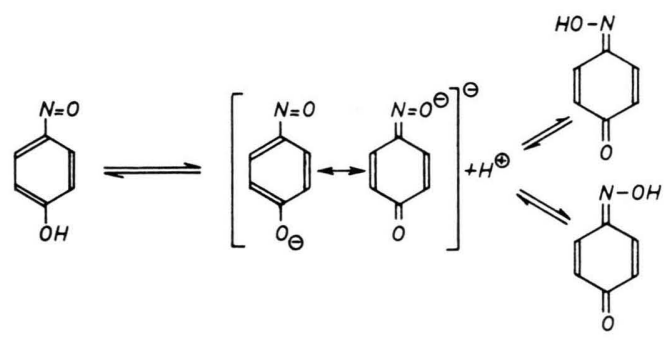

Die mesomere Grenzstruktur des NitrosophenolatIons erlaubt nicht nur eine schnelle Umorientierung zum Nitrosophenol, sondern auch der beiden OximKonformationen untereinander: Das NMR-Spektrum zeigt daher eine mittlere chemische Verschiebung für die Ringprotonen und scheinbare Äquivalenz der Protonen in 3- und 5-Stellung. In ätherischen Lösungsmitteln jedoch wird die mittlere Lebensdauer der nichtionogenen Konfigurationen erhöht. In Dioxan z. B. erscheint die Bande um 7,6 PPM ca. $40 \mathrm{~Hz}$ breit. Die Zugabe von wenig $\mathrm{H}^{\odot}$-Ionen ge-

* In dieser Arbeit beziehen sich alle Angaben der Chem. Verschiebung $\delta$ (PPM) auf Tetramethylsilan $=0$ (Innerer Standard). nügt nun, um das $\mathrm{AMXX}^{\prime}$-Spektrum des 1.4-Benzochinonoxims neben dem $\mathrm{AA}^{\prime} \mathrm{XX}^{\prime}$-Spektrum des 4Nitrosophenols zu erhalten (Abb. 1, Tab. 1). Die Integration ergibt bei $24{ }^{\circ} \mathrm{C} 85 \%$ Oxim neben $15 \%$ Nitrosophenol. Lösungen von 2.6-Dimethyl- und 2.6-Di-tert.butyl-benzochinon-4-oxim in Dioxan geben die erwarteten AM-Spektren für die Ringprotonen (Tab. 1) auch ohne Säurezugabe, ein Nitrosophenol-Anteil ist hier nicht mehr festzustellen. Die Chem. Verschiebung zwischen syn- und anti-Protonen liegt zwischen 0,5 und 0,6 PPM, erscheint also genügend groß für die Untersuchung der Raumisomerie.

Analoges gilt für unsymmetrisch substituierte Benzochinonoxime. Die Geschwindigkeit der Umorientierung in Aceton ist so groß, daß man vom 2-Chlor-benzochinon-4-oxim nur ein AMX-Spektrum erhält (Abb. 2 a). Die in Abb. 2 c angegebene $\mathrm{Zu}$ ordnung der beiden AMX-Spektren zur syn- und anti-Form wird später begründet. Der Anteil der Raumisomeren am Gleichgewicht ist bei $34{ }^{\circ} \mathrm{C} 65 \%$ syn $(\mathrm{Cl}), 35 \%$ anti $(\mathrm{Cl})$; ein Nitrosophenol-Anteil ist im Spektrum nicht erkennbar, muß also nach einer Abschätzung unter 5\% liegen.

Die Protonenresonanz ist also für die Untersuchung der Raumisomerie von Benzochinonoximen geeignet. Andererseits zeigt sich, daß die Raumisomeren in Lösung ein Gleichgewicht bilden, selbst bei nur geringer "Beteiligung“ der NitrosophenolatStruktur am mesomeren Ion. Die Isolierung beider Stereoisomeren aus der gleichen Lösung ist daher wahrscheinlich nicht möglich; es sei denn eine Dissoziation liege nicht vor, oder das Gleichgewicht sei stark temperaturabhängig.

11 E. Havinga u. A. Schors, Rec. Trav. chim. Pays-Bas 69, 457 [1950]. 


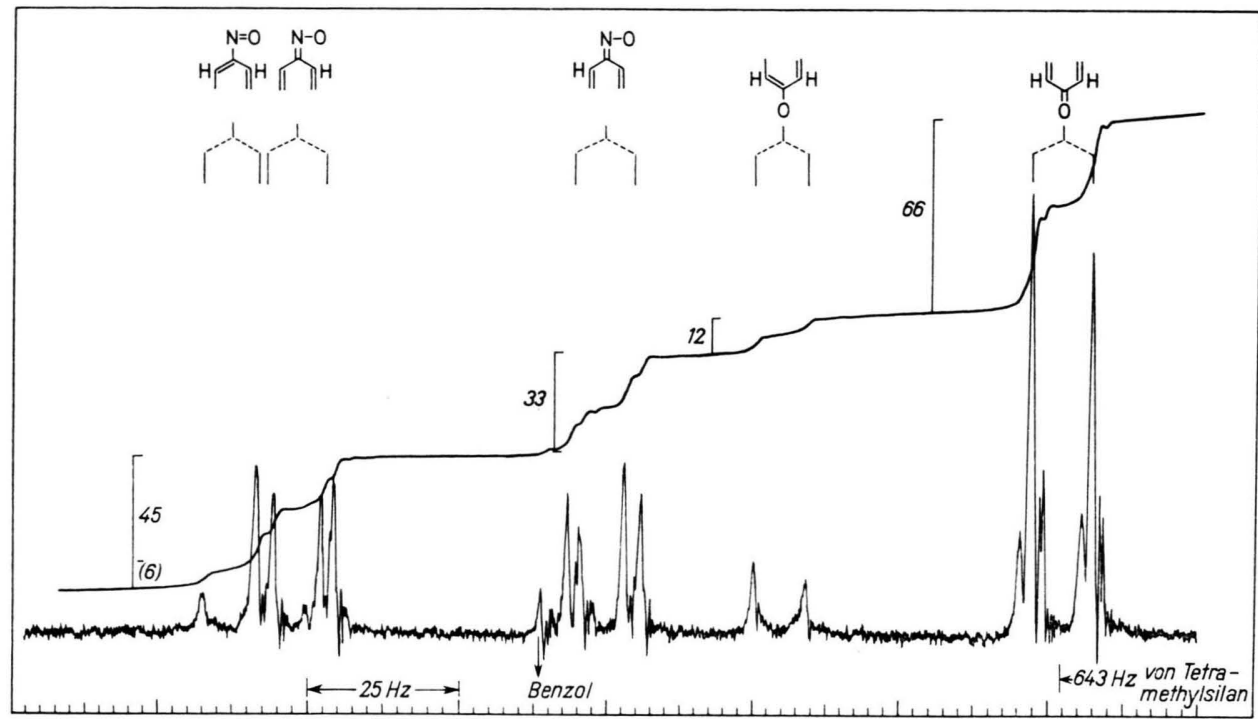

Abb. 1. 100-MHz-Protonenresonanz-Spektrum von 1.4-Benzochinonoxim $\rightleftarrows 4$-Nitrosophenol in Dioxan nach Zugabe von 3 Tropfen einer 5-proz. Lösung von Trifluoressigsäure in Tetrachlorkohlenstoff.

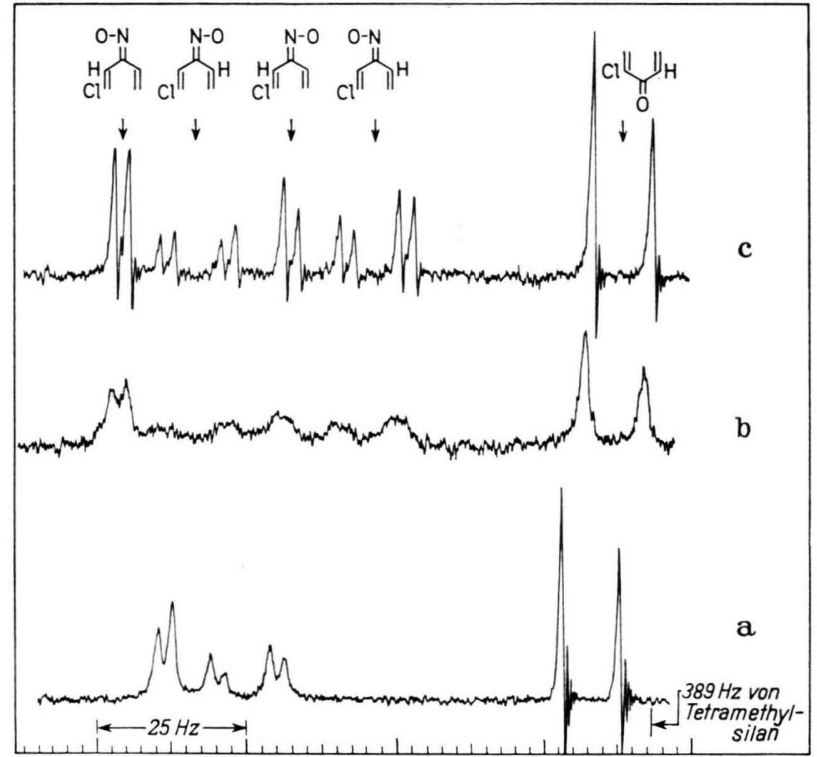

Abb. 2. 60-MHz-Protonenresonanz-Spektrum der Ringprotonen von 2-Chlorbenzochinon-4-oxim, a) in Aceton, b) in Dioxan, und c) in Dioxan nach Säurezugabe.

12 R. K. Norris u. S. Sternhell, Austral. J. Chem. 19, 841 [1966].

13 loc. cit. ${ }^{12}$, S. 851.

* Anm. b. d. Korr.: Während der redaktionellen Bearbeitung dieses Manuskripts haben R. K. Norris und S. Sternhell in "Tetrahedron Letters“ 1967, 97, nach Kenntnisnahme meiner 1. Mitt. ${ }^{1}$ ihre Formulierung „reguliert“" und die Annahme einer "Protonierung“ auf wasserhaltige Lösungen beschränkt. Außerdem geben sie weitere Gleichgewichtslagen von Raumisomeren-Gleichgewichten an. In beiden Mitteilungen ordnen übrigens die Autoren die Banden bei kleinerem Magnetfeld für die Protonen in 3-bzw. 5-Stel-
Kurz vor der ersten Mitteilung hierüber haben auch Norris und Sternhell ${ }^{12}$ eine Untersuchung über die Protonenresonanz des 1.4-Benzochinonoxims und einiger methylsubstituierter Chinonoxime veröffentlicht. Sie kommen im wesentlichen zu dem gleichen Ergebnis und erhalten Spektren vom Typ der Abbn. 1 oder $2 \mathrm{c}$ durch kurzzeitiges Erhitzen der Dioxan-Lösung auf $100{ }^{\circ} \mathrm{C}$ und anschließende Messung bei Sondentemperatur, allerdings - wie sie schreiben - unter „extremen Schwierigkeiten“. Die Zugabe von Säure - in Form wäßriger 3- $n$. Salzsäure - ergibt nach den Autoren wieder Spektren vom Typ der Abb. 2 a; sie nehmen daher auch kein Gleichgewicht im oben formulierten Sinne, sondern eine Protonierung des Oxims bzw. des Nitrosophenols an ${ }^{13}$. Nach meiner Erfahrung liegt die "Schwierigkeit" in der Zugabe wäßriger Lösung; die zu verstärkter Dissoziation führt. Drei Tropfen einer Tetrachlorkohlenstoff-Lösung von Chlorwasserstoff ergeben ohne weiteres Spektren vom Typ der Abbn. 1 oder $2 \mathrm{c}$, wenn das Dioxan wasserfrei und frei von größeren Mengen Peroxiden ist*.

lung der syn-Konfiguration zur Oximgruppe in 4-Stellung zu, ohne dafür andere Argumente anzugeben als „Analogie zu anderen Oximen und verwandten Verbindungen". Es ist wohl nicht überflüssig darauf hinzuweisen, daß die Verhältnisse bei Ketoximen oder Aldoximen nicht ohne weiteres auf Chinonoxime zu übertragen sind, zumal nach G. J. Karabatsos ${ }^{13}$ a syn-Protonen in entsprechenden Positionen z. B. von Aldoximen je nach Lösungsmittel stärker oder schwächer magnetisch abgeschirmt werden, als anti-Protonen.

13a G. J. Karabatsos u. Mitarb., J. Amer. chem. Soc. 85, 2326 [1963]. 


\section{B. Optische Bestimmung des Nitrosophenol-Anteils am Tautomeren-Gleichgewicht}

Eines der beiden AMX-Spektren in Abb. $2 \mathrm{c}$ könnte das des 2-Chlor-4-nitroso-phenols sein, wenn auch die Wahrscheinlichkeit dafür gering ist, denn für das Proton in 6-Stellung erwartet man in diesem Falle eine andere Chem. Verschiebung und andere Kopplungskonstanten als für das 6-Proton des Chinonoxims. Nach den Werten von Tab. 1 sollte das Proton beim 2-Chlor-4-nitrosophenol als Dublett mit $J \sim 9 \mathrm{~Hz}$ um 7 PPM liegen.

Immerhin empfiehlt sich doch eine unabhängige Bestimmung der Nitrosophenol-Beteiligung am Gleichgewicht. Hertel und LeboK ${ }^{14}$ haben zuerst die Messung der Nitroso-Lichtabsorptionsbande bei $750 \mathrm{~nm}-\mathrm{n} \rightarrow \pi^{*}$-Übergang der Nitroso-Gruppe ${ }^{15}$ - für die Untersuchung der Nitrosophenol-Tautomerie vorgeschlagen, da Chinonoxime in diesem Spektralgebiet nicht absorbieren. Da wegen der Gleichgewichtslagen die molaren Extinktions-Koeffizienten der Nitrosophenole nicht bekannt sind, nehmen die Autoren den Wert des 4-Nitrosoanisols $\varepsilon_{745}=57\left(\mathrm{l} \cdot \mathrm{mol}^{-1} \cdot \mathrm{cm}^{-1}\right)$ als Standard. Havinga ${ }^{16}$ bediente sich dieser Methode mit einem Standardwert $\varepsilon=45$ (Mittelwert einer Reihe aromatischer Nitrosoverbindungen). Hier stellt sich die Frage, ob es nicht doch ein „echtes“ 4-Nitrosophenol gibt, das auch in Lösungen keine oder nur geringe Neigung zur Oximbildung zeigt. Die orientierende Sichtung des vorhandenen Materials läßt dafür wenig Hoffnung. Die genauer untersuchten Verbindungen sind im festen Zustand Oxime ${ }^{2,3}$ und zeigen in Lösung nur geringe Neigung zur Nitrosophenol-Bildung ${ }^{16}$.

Es soll jedoch nicht unerwähnt bleiben, daß HodGson ${ }^{17}$ das 3-Fluor-4-nitrosophenol als dunkelgrüne Substanz beschreibt. Wegen der von ihm mitgeteilten merkwürdigen Eigenschaften wurde diese Verbindung hier nicht untersucht.

Durch IR-spektroskopische Untersuchungen ${ }^{18,19}$ ist jedoch bekannt, daß in Verbindungen wie dem Salicylsäuremethylester eine starke innere Brückenbindung des phenolischen Wasserstoffatoms an die Carbonestergruppe vorliegt, die in keinem der untersuchten Lösungsmittel eine Dissoziation dieses Wasserstoffatoms zuläßt. Der 5-Nitroso-salicylsäuremethylester (I) ist leicht zugänglich und wie erwartet eine reine, auch im festen Zustand monomere, Nitrosoverbindung. Das IR-Spektrum in Tetrachlorkohlenstoff zeigt unabhängig von der Konzentration nur eine für die innere Brückenbindung charakteristische schwache $\mathrm{OH}$-Bande um $3120 \mathrm{~cm}^{-1}$ (vgl. das Festkörperspektrum in Abb. 3).

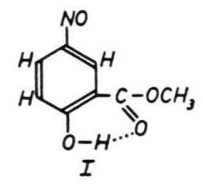

Die $\mathrm{N}=\mathrm{O}$-Lichtabsorptionsbande dieser Verbindung liegt in Aceton, Benzol und Dioxan bei $735 \mathrm{~nm}$ mit $\varepsilon=55$, unabhängig von der Konzentration in einem weiten Bereich und Temperatur-unabhängig von $20-60^{\circ} \mathrm{C}$. Da die Messung an reinem 4-Ni-

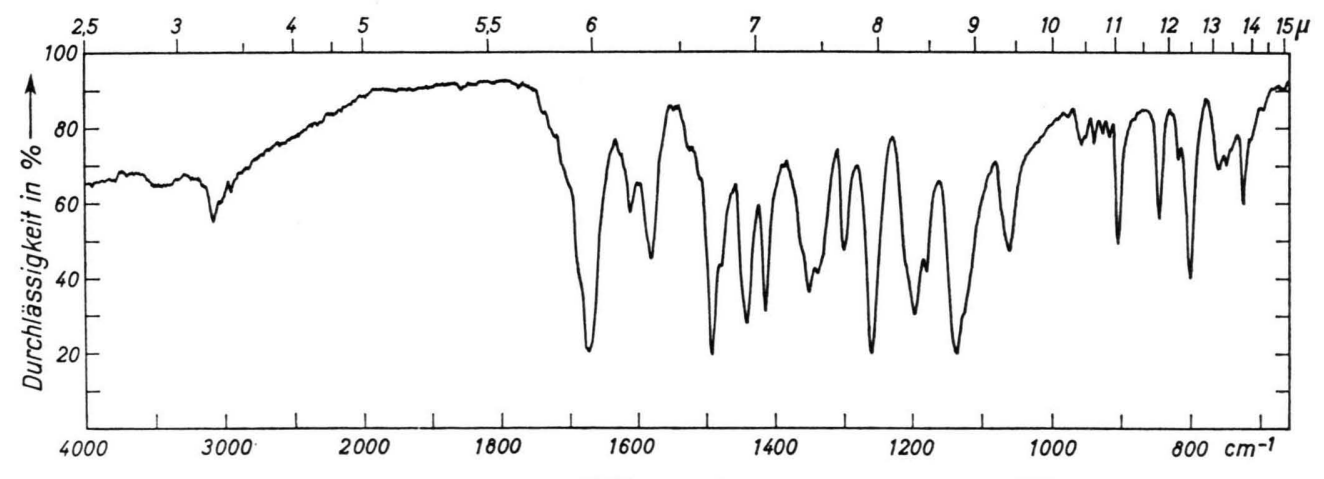

Abb. 3. IR-Spektrum von 5-Nitrososalicylsäuremethylester in $\mathrm{KBr}$.

14 E. Hertel u. F. Lebok, Z. physik. Chem. Abt. B 47, 315 [1940].

15 L. E. ORGEL, J. chem. Soc. [London] 1953, 1276.

16 A. Schors, A. Kraijeveld u. E. Havinga, Rec. Trav. chim. Pays-Bas 74, 1243 [1955].
17 H. H. Hodgson, J. chem. Soc. [London] 1940, 1268 u. 1943, 89.

18 W. Lüttke u. R. Mecke, Z. physik. Chem. 196, 56 [1951].

19 H. Hoyer, Z. physik. Chem. N.F. 36, 320 [1963]. 
trosoanisol in Benzol ebenfalls $\varepsilon_{745}=55$ ergab, ist dieser Wert für die Berechnung der NitrosophenolAnteile in der Tab. 2 benutzt worden.

\begin{tabular}{|c|c|c|c|c|}
\hline Chinon-4-oxim & Medium & $\begin{array}{c}c \\
{[\mathrm{~mol} / l]}\end{array}$ & $\begin{array}{l}\lambda_{\mathrm{No}} \\
{[\mathrm{nm}]}\end{array}$ & $\begin{array}{c}\text { Nitro- } \\
\text { sophenol } \\
{[\%]}\end{array}$ \\
\hline unsubstituiert & Aceton & $4,6 \cdot 10^{-3}$ & 736 & 25 \\
\hline unsubstituiert & Dioxan & $4,6 \cdot 10^{-3}$ & 738 & 14 \\
\hline 2.6-Dimethyl- & Dioxan & $4,8 \cdot 10^{-2}$ & - & $<0,2$ \\
\hline 2.6-Di-tert.butyl- & Dioxan & $3,7 \cdot 10^{-2}$ & - & $<0,2$ \\
\hline 2-Methyl- & Dioxan & $3,5 \cdot 10^{-2}$ & 738 & 1,5 \\
\hline 2-Chlor- & Aceton & $4,6 \cdot 10^{-2}$ & 738 & 3 \\
\hline 2-Chlor- & Dioxan & $4,6 \cdot 10^{-2}$ & 740 & 3 \\
\hline 2-Methoxy- & Dioxan & $4,7 \cdot 10^{-2}$ & 722 & $<1$ \\
\hline 2-Chlor-6-methyl & Dioxan & $5,3 \cdot 10^{-2}$ & 730 & $<0,5$ \\
\hline 3-Methyl- & Dioxan & $2,4 \cdot 10^{-2}$ & 758 & 3 \\
\hline 3-Methoxy- & Dioxan & $1,2 \cdot 10^{-2}$ & - & $<0,4$ \\
\hline 3-Chlor- & Dioxan & $2,4 \cdot 10^{-2}$ & 768 & 4 \\
\hline 3-Met.-6-tert.butyl- & Dioxan & $3,7 \cdot 10^{-2}$ & - & $<0,2$ \\
\hline 3-Methyl-6-isopropy- & Dioxan & $4,0 \cdot 10^{-2}$ & - & $<0,2$ \\
\hline 2.3.6-Trimethyl- & Dioxan & $4,8 \cdot 10^{-2}$ & - & $<0,2$ \\
\hline 3-tert. Butyl- & Dioxan & $4,0 \cdot 10^{-2}$ & 765 & 1 \\
\hline 3-Carbmethoxy- & Dioxan & $2,5 \cdot 10^{-2}$ & 740 & 22 \\
\hline 2-Chlor-5-methyl- & Dioxan & $3,5 \cdot 10^{-2}$ & 755 & $<1$ \\
\hline 2-Methyl-5-chlor- & Dioxan & $3,5 \cdot 10^{-2}$ & 745 & $<1$ \\
\hline 3,5-Dimethyl- & Dioxan & $2,7 \cdot 10^{-2}$ & 780 & 6 \\
\hline
\end{tabular}

Tab. 2. Nitrosophenolanteil in Lösungen von 1.4-Benzochinonoximen. Temperatur bei allen Messungen $20^{\circ} \mathrm{C}$.

Bei einer Reihe dieser Substanzen wurde die Konzentration zwischen $10^{-1}$ und $5 \cdot 10^{-3} \mathrm{Mol} / l$ variiert und keine Abhängigkeit des Extinktions-Koeffizienten von der Konzentration gefunden. Ebenso bleibt die Extinktion in allen Fällen nach Zugabe von Trifluoressigsäure zur Lösung unverändert. Außer beim 1.4-Benzochinonoxim selbst, dem Benzochinon-4oxim-3-carbonsäure-methylester und dem 3.5-Dimethyl-benzochinon-4-oxim ist der NitrosophenolAnteil zu gering, um bei unseren NMR-Spektren überhaupt nachgewiesen werden zu können. Der Wert für das 4-Nitrosophenol stimmt gut mit dem der NMR-Messung überein.

Damit besteht wohl kein Zweifel mehr, daß Abb. 2 c die beiden AMX-Spektren der raumisomeren 2-Chlor-benzochinon-4-oxime wiedergibt. Bemerkenswert ist noch, daß bei diesem Oxim auf optischem Wege der Nitrosophenol-Anteil in Aceton und in Dioxan gleich gefunden wird. Trotzdem erhält man das Spektrum der beiden Raumisomeren nur in Dioxan.

\section{Die Protonenresonanz-Spektren der in 3-Stellung substituierten Benzochinon-4-oxime}

Für die Aufklärung der Stereoisomerie fehlt nun noch eine Zuordnung der um ca. 0,5 PPM gegen- einander verschobenen Resonanzsignale der 3- und 5-Protonen zur $=\mathrm{N}$-O-syn- und anti-Konformation. Nachdem sich die Wirksamkeit der inneren Brückenbindung bei der Gewinnung eines 4-Nitrosophenols bewährt hatte, sollte versucht werden, ob vielleicht im Benzochinon-4-oxim-3-carbonsäure-methylester durch innere Brückenbindung eine Konformation (II) festgelegt ist, die eine Zuordnung des anti-Protons in 5-Stellung erlauben würde. Von der Raum-
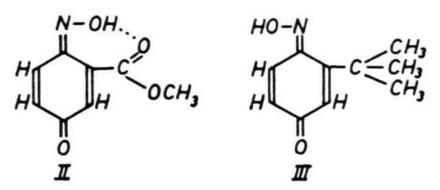

erfüllung einer tert. Butyl-Gruppe in 3-Stellung erhoffte ich andererseits eine ausschließliche Begünstigung der syn-Oxim-Konformation zum Proton in 5Stellung (III). Nach Überwindung einiger Schwierigkeiten gelang es, den Benzochinon-4-oxim-3-carbonsäuremethylester darzustellen, der jedoch außer einer starken Neigung zum Nitrosophenol keine Besonderheit gegenüber anderen in 3-Stellung substituierten Chinonoximen aufweist. Eine innere Brükkenbindung liegt nicht vor (IR-Spektrum in Abb. 8).

In Tab. 3 sind die Protonenresonanz-Spektren der in Nachbarstellung zur Oximgruppe substituierten 1.4-Benzochinonoxime zusammengefaßt. Die relativ große Zahl dieser Verbindungen hat sich aus einer ersten orientierenden Beobachtung ergeben, daß aus heißen Benzol-Lösungen von 3-Methyl-benzochinon4-oxim zunächst prismatische Kristalle, später filzige Nadeln fallen, und aus der Erwartung, daß bei diesen Oximen die Energiedifferenz der beiden Konfigurationen besonders groß sei. Der Energieunterschied reicht allerdings aus, daß bei normalen Temperaturen nur eines der beiden möglichen Stereoisomeren vorliegt, wie Tab. 3 zeigt. Für das Proton in 5-Stellung erhält man nur das Resonanzsignal bei kleinerem Magnetfeld. Alle Abweichungen von der Normallage 7,75 PPM - bei den Oximen \pm 0,25 PPM - sind durch den Einfluß der Substituenten, vornehmlich der in 6-Stellung bedingt. Da die Chem. Verschiebung dieser Resonanzabsorption praktisch unabhängig von Art und Größe der Substituenten in 3-Stellung ist, besteht wohl kein Zweifel, daß es sich um die des syn-Protons zur Oximgruppe handelt. Die Oximgruppe steht also in allen diesen Oximen anti zum Substituenten in 3Position, woraus sich die Zuordnung für die syn- 


\begin{tabular}{|c|c|c|c|c|c|}
\hline $\begin{array}{l}\text { Ringprotonen in Position } \\
\text { von Chinon-4-oximen }\end{array}$ & 5 & $\begin{array}{l}\delta \\
6\end{array}$ & 2 & $J_{56}$ & $J_{26}$ \\
\hline 3-Methyl- & 7,72 & 6,35 & 6,28 & 10,4 & 2,0 \\
\hline 3-Methyl-... methyläther & 7,60 & 6,33 & 6,28 & 10,5 & 0 \\
\hline 3-Methoxy- & 7,60 & 6,23 & 5,81 & 10,0 & 1,8 \\
\hline 3-Chlor- & 7,75 & 6,40 & 6,70 & 10,2 & 2,0 \\
\hline 3-Methyl-6-tert.butyl- & 7.59 & & 6,17 & & \\
\hline 3-Methyl-6-isopropyl- & 7,48 & & 6,22 & & \\
\hline 3-Methyl-6-isopropyl- . . methyläther & 7,38 & & 6,24 & & \\
\hline 3-tert.Butyl- & 7,85 & 6,35 & 6,30 & 10,7 & 1,9 \\
\hline 2.3.6-Trimethyl- & 7,53 & & & & \\
\hline 2.6-Dichlor-3-methyl- & 8,0 & & & & \\
\hline 3-Methoxycarbonyl & 7,78 & 6,47 & 6,61 & 10,4 & 1,9 \\
\hline 3-Methoxycarbonyl...methyläther & 7,67 & 6,45 & 6,60 & 10,2 & 1,9 \\
\hline
\end{tabular}

Tab. 3. Chemische Verschiebung $\delta(\mathrm{PPM})$ und Kopplungskonstanten $J(\mathrm{~Hz})$ von in 3-Stellung substituierten Benzochinon-4 oximen in Dioxan bei $34{ }^{\circ} \mathrm{C}$.

und anti-Protonen in 3- und 5-Stellung auch bei den anderen Chinonoximen ergibt.

Die beiden Kristallformen des 3-Methylbenzochinon-4-oxims wurden übrigens isoliert. Sie geben im kristallinen Zustand identische IR-Spektren und in Lösung beide das in Tab. 3 aufgeführte NMRSpektrum; bis $-50{ }^{\circ} \mathrm{C}$ in Tetrahydrofuran und bis $+90^{\circ} \mathrm{C}$ in Dioxan ist im Spektrum kein Anteil des anderen Raumisomeren bemerkbar.

2.3.6-Trimethyl-benzochinon-4-oxim zeigt kaum Neigung zur Nitrosophenol-Bildung und ist thermisch sehr stabil; es eignet sich daher besonders gut für die Suche nach anderen Stereoisomeren, die jedoch in Dioxan bis $+90^{\circ} \mathrm{C}$ bzw. in Cyclohexanon bis $+130{ }^{\circ} \mathrm{C}$ erfolglos blieb. Beide Raumisomere sind daher wahrscheinlich nur von Chinon-4-oximen zu erwarten, die nicht in einer Nachbarstellung zur Oximgruppe substituiert sind.

\section{Die Protonenresonanz-Spektren der in 2-Stellung substituierten Benzochinon-4-oxime und der stereo- isomeren Oximäther}

In Tab. 4 sind die Chem. Verschiebungen der Raumisomeren von den in 2-, oder in 2- und 6-Stellung substituierten Chinon-4-oximen zusammengefaßt. Nach den in Abschnitt A mitgeteilten Ergebnissen muß man in Lösung für die Oxime ein Gleichgewicht der Raumisomeren annehmen, dessen Temperaturabhängigkeit jedoch, wie Tab. 5 zeigt, gering ist.

\begin{tabular}{|c|c|c|c|c|c|}
\hline $\begin{array}{l}\text { Ringprotonen in Position } \\
\text { von Chinon-4-oximen }\end{array}$ & 3 & $\begin{array}{l}\delta \\
5\end{array}$ & 6 & $J_{56}$ & $J_{35}$ \\
\hline syn-2-Methyl- & 7,56 & 7,14 & 6,40 & 10,0 & 2,6 \\
\hline syn-2-Methyl- . . methyläther * & 7,42 & 7,08 & 6,40 & 10,1 & 2,7 \\
\hline anti-2-Methyl- & 7,04 & 7,68 & 6,40 & 10,0 & 2,4 \\
\hline anti-2-Methyl-...methyläther * & 6,96 & 7,53 & 6,40 & 10,1 & 2,5 \\
\hline syn-2-Chlor- & 7,95 & 7,25 & 6,56 & 10,0 & 2,5 \\
\hline syn-2-Chlor- . . methyläther & 7,85 & 7,20 & 6,55 & 10,0 & 2,5 \\
\hline anti-2-Chlor- & 7,50 & 7,75 & 6,56 & 10,0 & 2,5 \\
\hline anti-2-Chlor-. . . methyläther & 7,45 & 7,62 & 6,55 & 10,0 & 2,5 \\
\hline syn-2-Methoxy- & 6,81 & 7,14 & 6,44 & 10,0 & 2,3 \\
\hline anti-2-Methoxy- & 6,39 & 7,72 & 6,44 & 10,0 & 2,3 \\
\hline syn-2-Chlor-6-methyl- & 7,88 & 7,05 & & & 2,4 \\
\hline syn-2-Chlor-6-methyl-...methyläther & 7,78 & 7,01 & & & 2,5 \\
\hline anti-2-Chlor-6-methyl- & 7,39 & 7,55 & & & 2,5 \\
\hline anti-2-Chlor-6-methyl-... methyläther & 7,35 & 7,43 & & & 2,5 \\
\hline
\end{tabular}

Tab. 4. Chemische Verschiebung $\delta(\mathrm{PPM})$ und Kopplungskonstanten $J(\mathrm{~Hz})$ von in 2-Stellung substituierten Benzochinon-4oximen in Dioxan bei $34{ }^{\circ} \mathrm{C}$.

* Der 2-Methyl-1.4-benzochinon-4-oximmethyläther wurde nicht in die beiden raumisomeren Formen getrennt. 


\begin{tabular}{lcc}
\hline \multicolumn{1}{c}{ Benzochinon-4-oxim } & $t\left[{ }^{\circ} \mathrm{C}\right]$ & $\begin{array}{c}\text { Anteil } \\
{[\%]}\end{array}$ \\
\hline syn-2-Methyl- & 34 & 65 \\
syn-2-Chlor- & 24 & 65 \\
syn-2-Chlor- & 60 & 60 \\
syn-2-Methoxy- & 24 & 90 \\
syn-2-Methoxy- & 80 & 85 \\
syn-2-Chlor-6-methyl- & 34 & 50 \\
\hline
\end{tabular}

Tab. 5. Anteil der syn-Form am Raumisomeren-Gleichgewicht in Dioxan bei verschiedenen Temperaturen.

Auch die Temperaturabhängigkeit des Gleichgewichts 1.4-Benzochinonoxim $\rightleftarrows$ Nitrosophenol ist gering; in Benzol wurde im Temperaturbereich 20 bis $66{ }^{\circ} \mathrm{C}$ eine Umlagerungsenergie von 1,5 kcal/Mol gefunden.

Bei der Kristallisation des 2-Chlor-benzochinon-4oxims aus Chloroform/Petroläther $(3: 1)$ erhält man bei allmählich sinkender Temperatur zunächst Blättchen, die sich haufenweise sternförmig aneinanderlegen, später - ab ca. $0^{\circ}$ - nur lange spießige Nadeln. Aus Essigester fallen nur Blättchen, Schmp. $146^{\circ}$ (Zers.), aus Dioxan nur sechskantige prismatische Säulen, Schmp. $141^{\circ}$ (Zers.). Alle Formen ergeben jedoch im festen Zustand identische IRSpektren und in Lösung - wie erwartet - das NMR-Spektrum des Isomeren-Gleichgewichts. Das gleiche gilt für die Kristallformen des 2-Chlor-6methyl-benzochinon-4-oxims tafelförmige Blättchen, Schmp. $157^{\circ}$ (Zers.), und Nadeln, Schmp. $154^{\circ}$ (Zers.). Wahrscheinlich liegt Polymorphie vor. Die Aussichten dafür, daß die Stereoisomeren getrennt kristallisieren, sind also gering. Die letzte Entscheidung liegt hier jedoch bei der Diffraktionsanalyse.

Anders verhält es sich mit den Oximäthern. Die beiden bereits von Kenrmann getrennten Kristallformen des 2-Chlor-benzochinon-4-oximmethyläthers ${ }^{20}$ sind die Stereoisomeren. Kehrmanns „a-Form“, hellgelbe fasrige Nadeln, Schmp. $122-123{ }^{\circ} \mathrm{C}$, hat die Oximgruppe syn zum Chlor (Abb. 4a), die , $\beta$ Form“, gelbe Prismen, Schmp. $114-115{ }^{\circ} \mathrm{C}$, hat die Oximgruppe anti zum Chlor (Abb. 4 b). In Dioxan bis $+90{ }^{\circ} \mathrm{C}$ wandelt sich keines der beiden Raumisomeren in das andere um.

Der 2-Chlor-6-methyl-benzochinon-4-oximmethyläther läßt sich - wenn auch schwieriger - ebenfalls in die beiden Stereoisomeren zerlegen. Durch fraktionierte Kristallisation aus Benzin erhält man zunächst den anti-2-Chlor-6-methyl-benzochinon-4-

20 F. Kehrmann, Liebigs Ann. Chem. 279, 24 [1894].

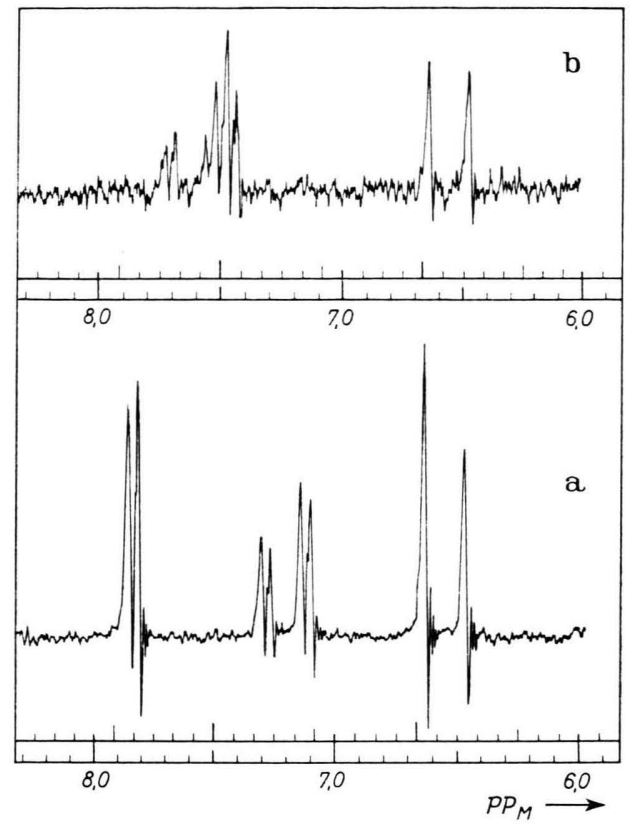

Abb. 4. 60-MHz-Protonenresonanz-Spektren von a) syn-2Chlor-benzochinon-4-oximmethyläther und b) anti-2-Chlorbenzochinon-4-oximmethyläther in Dioxan.

oximmethyläther, gelbe Blättchen, Schmp. $113{ }^{\circ} \mathrm{C}$, dann den leichter löslichen syn-2-Chlor-6-methylbenzochinon-4-oximmethyläther, gelbe Nadeln,Schmp. $78{ }^{\circ} \mathrm{C}$. Eine Umwandlung einer Form in die andere wurde auch bei längerem Erhitzen der Dioxanlösungen auf $100{ }^{\circ} \mathrm{C}$ nicht beobachtet.

\section{E. Die Zuordnung der Chem. Verschiebungen und die Größe der Spin-Spin-Kopplungskonstanten}

Die Stellung der Oximgruppe zu den Substituenten bei den bisher beschriebenen Verbindungen ergibt sich aus der Synthese; mit einer Ausnahme wurden alle durch Nitrosierung der Phenole dargestellt. Der Einfluß der anderen Substituenten auf die magnetische Abschirmung der Ringprotonen ist gleich dem in entsprechenden Systemen ${ }^{21}$. Alle Abweichungen der Chem. Verschiebung für die Protonen in 3- und 5-Stellung ergeben sich daraus. Die unter den Ringprotonen auftretenden Kopplungskonstanten variieren nur geringfügig; sie entsprechen den „ortho“- und „meta“-Kopplungskonstanten am Benzolring. Zahl und Größe der jeweils auftretenden Kopplungskonstanten erlauben daher eine

21 Vgl. z. B. L. M. Jackman, Application of Nuclear Magnetic Resonance in Organic Chemistry, London 1959. 
Zuordnung der Banden zu den einzelnen Protonen. Die in den Tabellen nicht aufgeführten WeitdistanzKopplungskonstanten $J_{(\mathrm{H}-\mathrm{C}=\mathrm{C}-\mathrm{CH} 3)}$ zwischen Ringprotonen und Protonen der Seitenketten betragen 1,0-1,6 Hz. Praktisch wurden nur Hyperfeinstruktur-Aufspaltungen 1. Ordnung gefunden, außer beim 4-Nitrosoanisol, beim 1.4-Benzochinonoxim und dessen Methyläther. Bei der letztgenannten Verbindung wurde die Zuordnung durch Spin-Spin-Entkopplung überprüft.

Im $60 \mathrm{MHz}$-Spektrum überlappen sich Banden des Nitrosophenols mit denen des Chinonoxims. Hier wie bei ähnlichen Überlagerungen in den Spektren von Stereoisomeren wurde die Zuordnung mit $100 \mathrm{MHz}-$ Spektren kontrolliert.

\section{F. Die „stabilen Stereoisomeren“ des 2-Chlor-5-methyl-benzochinon-4-oxims}

Kehrmann ${ }^{6}$ hat dieses Oxim in eine schwerer lösliche „ $\alpha$-Form“, Schmp. $170^{\circ} \mathrm{C}$, und eine leichter lösliche „ $\beta$-Form“, Schm. $165^{\circ} \mathrm{C}$, getrennt. Das wegen der Darstellung aus dem Chinon und Hydroxylamin grundsätzlich mögliche Vorliegen des 2-Methyl5-chlor-benzochinon-4-oxims meinte Kenrmans dadurch ausgeschlossen zu haben, daß er nach der Oxydation bzw. Reduktion beider Formen nur 4. Nitro-bzw. 4-Amino-2-chlor-5-methylphenol isolierte. Als weitere Stütze dafür, daß hier Stereoisomere vorliegen, sah Kehrmann Umwandlungen in 2 Acetylderivate an, die hier schematisch wiedergegeben werden sollen:

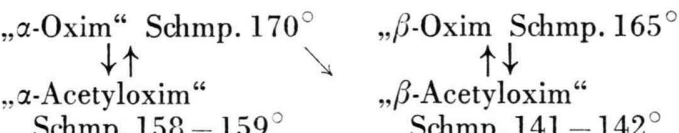

$$
\begin{aligned}
& \text { Schmp. } 158-159^{\circ} \quad \text { Schmp. } 141-142^{\circ}
\end{aligned}
$$

Niederländische Forscher haben sich eingehend mit diesem Oxim befaßt ${ }^{3,22-24}$ und die Ergebnisse von Kehrmann bestätigt, außer der von Kehrmann berichteten Umwandlung der „, $\beta$-Form“ in die , $\alpha$ Form“. Nach der Röntgendiffraktions-Analyse ist

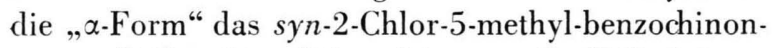
4 -oxim ${ }^{23}$. Für die , $\beta$-Form“ konnte die Diffraktionsanalyse nicht völlig exakt durchgeführt werden ${ }^{24}$; dennoch glauben die Autoren auf Grund ihrer Daten, des IR-Spektrums ${ }^{22}$ und der chemischen Befunde, daß hier das anti-2-Chlor-5-methyl-benzochinon-4-oxim vorliegt.

22 A. J. H. Umans, Dissertation, Leyden 1959.

23 C. Romers u. E. Fischmans, Acta crystallogr. [Copenhagen] 13,809 [1960].
Diese Ergebnisse sind nicht gut mit den in $\mathrm{Ab}$ schnitt A mitgeteilten zu vereinbaren, sie stehen aber im klaren Widerspruch zu den Ergebnissen von Abschnitt C, wonach hier nur ein Stereoisomeres, das mit der Oxim-Gruppe syn zum Chlor vorliegen soll.

Wir haben daher die Versuche wiederholt. Die IR-Spektren der von uns gefundenen „ $\alpha$-Form“, goldgelbe Nadeln, Schmp. $194{ }^{\circ} \mathrm{C}$ (Zers.), und der „ $\beta$-Form“, grauweiße filzige Kriställchen, Schmp. $185^{\circ} \mathrm{C}$ (Zers.), stimmen völlig mit den von Umans ${ }^{22}$ angegebenen überein. Die „ $\alpha$-Form“ ist, wie erwartet, das syn-2-Chlor-5-methyl-benzochinon-4-oxim (A) (Tab. 6). Abb. 5 zeigt das ProtonenresonanzSpektrum der , $\beta$-Form“. Neben den Banden der „ $\alpha$ Form finden sich solche bei 7,57 (Kopplung von

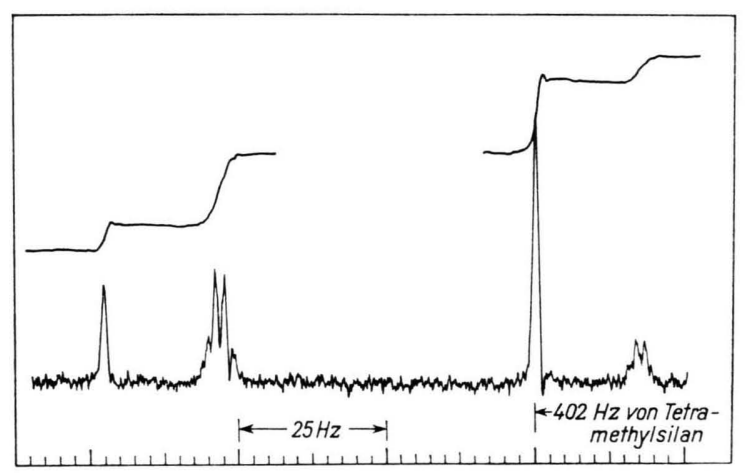

Abb. 5. 60-MHz-Protonenresonanz-Spektrum des Adduktes von 2-Chlor-5-methyl- mit dem 2-Methyl-5-chlor-benzochinon4-oxim in Dioxan.

1,4 $\mathrm{Hz}$ mit den Protonen einer Methylgruppe) und 6,69 PPM. Nach der Chem. Verschiebung und der Kopplungskonstanten und unter Berücksichtigung der Elementarzusammensetzung kann es sich nur um die Banden des syn-2-Methyl-5-chlor-benzochinon-4oxims handeln (B) (Tab. 6) .

\begin{tabular}{lcccc}
\hline $\begin{array}{l}\text { Ringprotonen in Position } \\
\text { von Chinon-4-oximen }\end{array}$ & 3 & 6 & $J_{\left(\mathrm{H}-\mathrm{C}=\mathrm{C}-\mathrm{CH}_{3}\right)}$ \\
\hline 2-Chlor-5-methyl- & 7,90 & 6.40 & 1,4 \\
2-Methyl-5-chlor- & 7,57 & 6,69 & 1.4 \\
2-Chlor-5-methyl-... acetat & 7,87 & 6,53 & 1,4 \\
2-Methyl-5-chlor-... acetat & 7,54 & 6,85 & 1,4 \\
\hline
\end{tabular}

Tab. 6. Chemische Verschiebung $\delta$ (PPM) und Kopplungskonstanten $J(\mathrm{~Hz})$ von 2.5-disubstituierten Benzochinon-4. oximen in Dioxan.

24 E. Fischmanx, C. Romers u. A. J. H. Umans, Acta crystallogr. [Copenhagen] 13, 885 [1960]. 
Beide Strukturisomere bilden also in der sogenannten „ $\beta$-Form“ ein Addukt. Nach der Umkristallisation aus Toluol ist das Verhältnis $\mathrm{A} / \mathrm{B}=1: 4$; das Addukt hat jedoch noch Lösungsmittel eingeschlossen. Nach der Sublimation dieses Präparates erhält man $\mathrm{A} / \mathrm{B}=2: 5$. Selbstverständlich wurde nun auch das 2-Methyl-5-chlor-benzochinon-4-oxim ${ }^{25}$ durch Nitrosierung des Phenols dargestellt (NMRSpektrum in Tab. 6). 1,5 g dieses Oxims mit 0,4 $\mathrm{g}$ des Strukturisomeren zusammen aus Toluol umkristallisiert ergab die „ $\beta$-Form“ Kehrmanns mit einem Verhältnis der Strukturisomeren $\mathrm{A} / \mathrm{B}=1: 3$. Die Adduktbildung ist also in einem weiten Mischungsbereich möglich. Das IR-Spektrum des Addukts ist aus den Spektren der Strukturisomeren zusammensetzbar außer einer intensiven Bande bei $1055 \mathrm{~cm}^{-1}$; die Bande des 2-Chlor-5-methyl-benzochinon-4-oxims liegt bei $1039 \mathrm{~cm}^{-1}$, die des 2-Methyl-5-chlor-benzochinon-4-oxims bei $1052 \mathrm{~cm}^{-1}$. Auf die sonstigen interessanten Eigenschaften des Addukts kann hier nicht eingegangen werden ${ }^{24}$. Die Acetyl-Derivate Kehrmanss sind in mehr oder weniger großem Umfang Gemische des syn-2-Chlor-5-methyl-benzochinon-4-oxim-acetats, Schmp. $161{ }^{\circ} \mathrm{C}$, und des syn2-Methyl-5-chlor-benzochinon-4-oxim-acetats, Schmp. $153{ }^{\circ} \mathrm{C}$ (NMR-Spektren in Tab. 6). Das „a-Oxim“ Kehrmanns war noch nicht frei vom Strukturisomeren.

Es besteht wohl kein Zweifel, daß für die hier nicht untersuchten entsprechend bromsubstituierten Oxime das gleiche gilt.
G. Das Protonenresonanz-Spektrum des

\section{5-Dimethyl-benzochinon-4-oxim-methyläthers}

In diesem Zusammenhang interessiert noch die Anordnung der Oximgruppe, wenn beide benachbarte Positionen substituiert sind. Das Spektrum des 3.5-Dimethyl-benzochinon-4-oxims konnten wir nicht erhalten, teils wegen der geringen Löslichkeit, teils wegen der Tendenz zur Nitrosophenolbildung. Abb. 6 zeigt jedoch das Spektrum des Methyläthers, wobei interessant ist, daß nicht nur die Methylgruppen, sondern auch die 2.6-Ringprotonen unterschiedlich abgeschirmt sind, was bisher bei keinem Chinonoxim beobachtet wurde. Die Chem. Verschiebung zwischen den Ringprotonen ist durch ein $100 \mathrm{MHz}$ Spektrum gesichert.

Von diesem Typ müssen zunächst weitere Verbindungen dargestellt und untersucht werden, bevor Aussagen über die Anordnung der Oxim-Gruppe gemacht werden können.

\section{H. Darstellung der Verbindungen}

Wegen der Fülle des Materials kann hier die Darstellung und Reinigung der meisten Oxime nicht im einzelnen beschrieben werden; die Mehrzahl ist auch bereits bekannt. Teilweise wurden die Phenole nach anderen Verfahren nitrosiert (Tab. 7), als in der Literatur angegeben. Am meisten bewährt hat sich das Eintragen von festem Natriumnitrit in Lösungen der Phenole in alkoholisch-wäßriger Salzsäure (Ver-

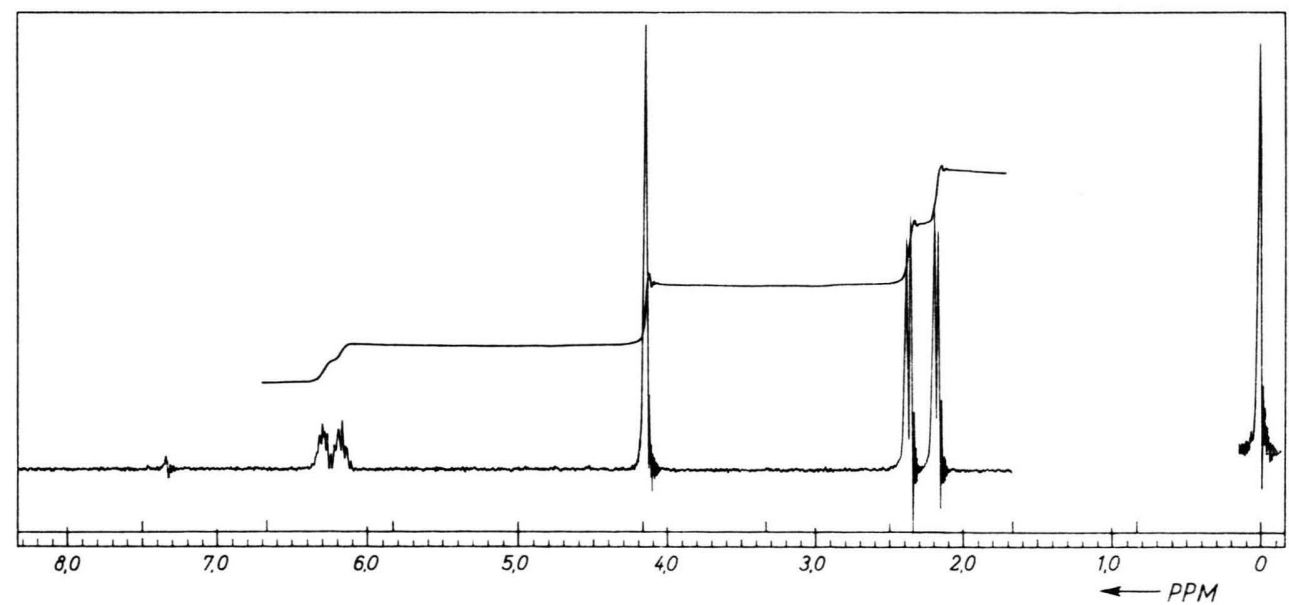

Abb. 6. 60-MHz-Protonenresonanz-Spektrum des 3.5-Dimethyl-benzochinon-4-oxim-methyläthers in Deuterochloroform.

25 H. H. Hodgson u. F. Moore, J. chem. Soc. [London] 1926. 2037. 


\begin{tabular}{|c|c|c|c|}
\hline Chinon-4-oxim & $\begin{array}{l}\text { Verfahren der } \\
\text { Darstellung }\end{array}$ & $\begin{array}{c}\text { Umkristallisiert } \\
\text { aus }\end{array}$ & $\begin{array}{c}\text { Schmp. } \\
{[\%]}\end{array}$ \\
\hline unsubstituiert & $\mathrm{C}$ & Benzol & $130^{\circ}$ Zers \\
\hline 2.6-Dimethyl- & $\mathrm{A}$ & Tetrachlorkohlenstoff & $170^{\circ}$ Zers \\
\hline 2.6-Di-tert.butyl- & $\mathrm{A}$ & Benzin & $215^{\circ}$ Zers \\
\hline 2-Methyl- & $\mathrm{C}$ & Benzol & $135^{\circ}$ Zers \\
\hline 2-Chlor- & $\mathrm{C}$ & Chloroform & $147^{\circ}$ Zers \\
\hline 2-Methoxy- & A & Essigester & $174^{\circ}$ Zers \\
\hline 2-Chlor-6-methyl- & $\mathrm{A}$ & Methanol & $155^{\circ}$ Zers \\
\hline 3-Methyl- & A & Benzol & $159^{\circ}$ Zers \\
\hline 3-Methoxy- & 30 & Essigester & $175^{\circ}$ Zers \\
\hline 3-Chlor- & B & Methanol/Wasser & $184^{\circ}$ Zers \\
\hline 3-Methyl-6-tert.butyl- & A & Benzol & $180^{\circ}$ Zers \\
\hline 3-Methyl-6-isopropyl- & A & Chloroform & $170^{\circ}$ Zers \\
\hline 3-tert.Butyl- & B & Methanol/Wasser & $176^{\circ}$ Zers \\
\hline 2.3-6-Trimethyl- & 31 & Wasser & $181^{\circ}$ \\
\hline 2-Chlor-5-methyl- & 6 & Toluol & $194^{\circ}$ Zers \\
\hline 2-Methyl-5-chlor- & A & Benzol & $182^{\circ}$ Zers \\
\hline 3,5-Dimethyl- & A & Methanol & $178^{\circ}$ Zers \\
\hline
\end{tabular}

Tab. 7. Darstellungsverfahren und Schmelzpunkte der 1.4-Benzochinonoxime.

fahren A) ${ }^{26}$; in anderen Fällen ist die Umsetzung mit Nitrosylschwefelsäure ${ }^{27}$ zweckmäßig (Verfah ren $B$ ) oder die Methode von BRIDGe ${ }^{28}$ (Verfahren C). Bei den Nitrosierungen entstehende Nebenprodukte wie Diphenochinone und Chinondoazide sind durch ihre Elementarzusammensetzung und durch ihr IR-Spektrum (Chinondiazide absorbieren z. B. bei $2150 \mathrm{~cm}^{-129}$ ) erkennbar.

Der 1.4-Benzochinonoxim-methyläther, Schmp. 83 bis $84{ }^{\circ} \mathrm{C}$, wurde aus Chinon und $O$-Methylhydroxylamin ${ }^{32}$, alle in Tab. 8 aufgeführten Oximäther aus den Oximen mit Dimethylsulfat ${ }^{32}$ hergestellt.

\begin{tabular}{lc}
\hline Chinon-4-oximmethyläther & $\begin{array}{c}\text { Schmp. } \\
{\left[{ }^{\circ} \mathrm{C}\right]}\end{array}$ \\
\hline 2-Methyl- & 73,5 \\
syn-2-Chlor- & 122,5 \\
anti-2-Chlor- & 114,5 \\
syn-2-Chlor-6-methyl- & 78 \\
anti-2-Chlor-6-methyl- & 112,5 \\
3-Methyl- & 73 \\
3-Methyl-6-isopropyl- & 34 \\
3.5.-Dimethyl- & 112,5
\end{tabular}

Tab. 8. Schmelzpunkte von Chinonoximäthern.

Die Trennung der stereoisomeren 2-Chlor-6-methyl-benzochinon-4-oxim-methyläther erfolgte durch fraktionierte Kristallisation aus Benzin zwischen

26 Organic Syntheses, Coll. Vol. I, 511, New York 1958.

27 H. H. Hodgson u. D. E. Nicholson, J. chem. Soc. [London] 1939, 1808.

28 J. L. Bridge, Liebigs Ann. Chem. 277, 85 [1839].

29 J. W. Le Fevre u. Mitarb., J. chem. Soc. [London] 1954 , 4686 .
+30 und $-35^{\circ} \mathrm{C}$. Zunächst fallen die schwerer löslichen gelben Blättchen, Schmp. $112,5^{\circ} \mathrm{C}$; mit sinkender Temperatur der Lösung wird der Anteil der leichter löslichen gelben Nadeln größer. Bei $-35{ }^{\circ} \mathrm{C}$ fallen schließlich fast reine Nadeln, Schmp. $78^{\circ} \mathrm{C}$, die nur noch $5 \%$ des anderen Stereoisomeren enthalten. Die Trennung der stereoisomeren 2-Chlorbenzochinon-4-oximmethyläther ist bei Kenrmann ${ }^{20}$ beschrieben.

Die Acetyl-Derivate des 2-Chlor-5-methyl- und des 2-Methyl-5-chlor-benzochinon-4-oxims erhielten wir nach dem von KehrmanN ${ }^{6}$ beschriebenen Verfahren.

Die Darstellung des 1.4-Benzochinon-4-oxim-3carbonsäure-methylesters war mit Schwierigkeiten verbunden. Da außerdem diese Struktur bereits von GibBs ${ }^{33}$ für ein kaum lösliches amorphes, braunes Pulver beansprucht wurde, das er aus dem Gentisinsäuremethylester, Wasserstoffperoxid und Hydroxylamin erhalten hat, soll die Darstellung dieser Verbindung genauer beschrieben werden. Die von GibBs ebenfalls diskutierte Formel eines 2-Methoxycarbonyl-4-oxims entfällt, da dieser Struktur der in Abschnitt B erwähnte 5-Nitroso-salicylsäure-methylester entspricht. Sind schon die von GiBBs angegebenen Eigenschaften des amorphen Pulvers wenig vertrauenerweckend, so zeigt das IR-Spektrum einer

${ }^{30}$ F. Henrich u. B. Rhodius, Ber. dtsch. chem. Ges. 35, 1475 [1902].

31 R. Nietzki u. J. Schneider, Ber. dtsch. chem. Ges. 27, 1430 [1894].

32 S. Veibel u. M. Hejde-Simesen, Ber. dtsch. chem. Ges. 63, 2476 [1930].

33 H. D. Gibis, J. Amer. chem. Soc. 34, 1202 [1912]. 
nach seiner Vorschrift hergestellten Probe ${ }^{33}$, daß es sich wohl um ein Gemisch verschiedener Verbindungen handelte, unter denen die gesuchte nicht vorliegt. Das gesuchte Produkt war weder durch Umsetzung des Chinoncarbonsäureesters mit Hydroxylamin noch durch Nitrosierung des $m$-Oxybenzoesäureesters zu erhalten. Bei der mit allen Varianten versuchten Nitrosierung findet entweder keine Re- aktion statt oder es bilden sich unter forcierten Bedingungen 2-Nitro- und 4-Nitrophenol-5-carbonsäuremethylester.

Eine Übertragung der von Ciamician und Silber ${ }^{34}$ entdeckten photochemischen Umlagerung von $o$ Nitrobenzaldehyd führte schließlich auf zwei Wegen zur gewünschten Verbindung:

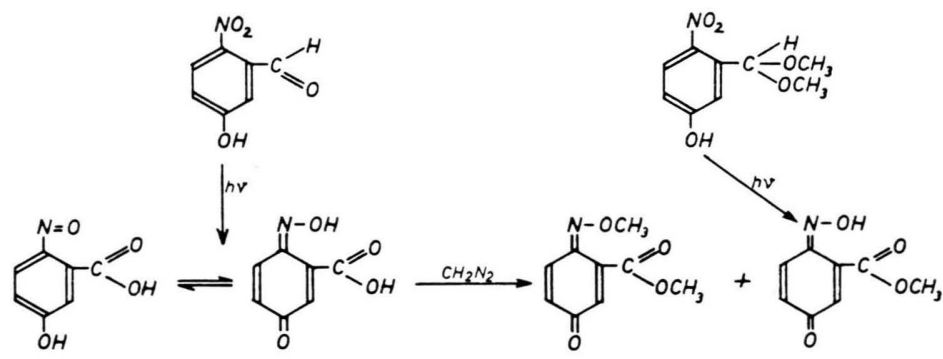

Wie wir heute wissen, verläuft die Reaktion des Nitrobenzaldehyds bei kurzer Lichteinwirkung deswegen so eindeutig zu der Nitrosoverbindung, weil deren Dimeres schwer löslich ist und auskristallisiert. Bei längerer Lichteinwirkung entstehen zahlreiche Folgeprodukte. Es galt also, ein Medium zu finden, aus dem das gewünschte Reaktionsprodukt möglichst bald kristallisiert, was nach einigem Experimentieren gelang. Der 1.4-Benzochinon-4-oxim3 -carbonsäure-methylester ist sehr wasserempfindlich, was zunächst einige Schwierigkeiten bereitete.

Die 5-Nitroso-salicylsäure ist bekannt ${ }^{35,36}$; mit Diazomethan erhält man daraus leicht den Methyl- ester, der bereits in geringer Menge von Houben ${ }^{37}$ erhalten wurde.

\section{Experimentelles}

\section{5-Nitrosalicylsäuremethylester}

5-Nitrosalicylsäure wird in Äther gelöst und bei $-5{ }^{\circ} \mathrm{C}$ mit der äquivalenten Menge Diazomethan in Äther umgesetzt. Der Äther wird verdampft und der Rückstand zweimal aus Benzin umkristallisiert. Türkisfarbene Kristalle, Schmp. $90-91{ }^{\circ} \mathrm{C}$. Ausbeute: $50 \%$ d. Theorie. Gefunden: C 53,1, H 4,1\%; berechnet für $\mathrm{C}_{8} \mathrm{H}_{7} \mathrm{NO}_{4}$ : C 53,0, $\mathrm{H} \mathrm{3,9}$ Prozent.

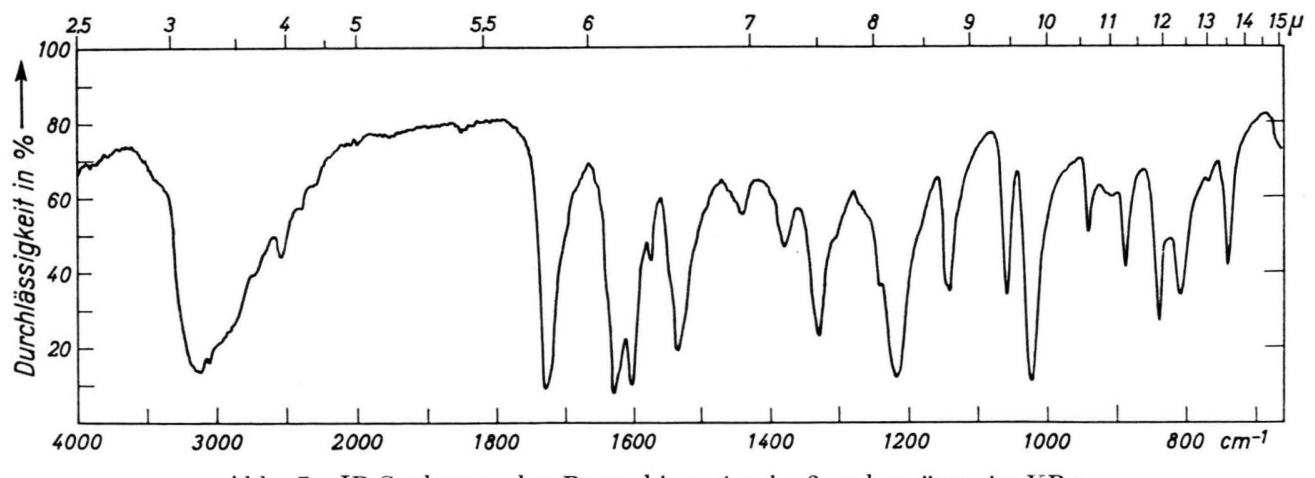

Abb. 7. IR-Spektrum der Benzochinon-4-oxim-3-carbonsäure in $\mathrm{KBr}$.

34 G. Ciamician u. P. Silber, Ber. dtsch. chem. Ges. 34, 2040 [1901]; 35, 1992 [1902].

35 W. Gulinow, J. chem. Ind. (russ.) 5, 225 ; C. 1928 II, 759.
36 A. A. Nemodruk, J. allgem. Chem. (russ.) 28 (90), 1082 [1958]; C. 1960, 5142 .

37 J. Houben u. G. Schreiber, Ber. dtsch. chem. Ges. 53. 2356 [1920]. 


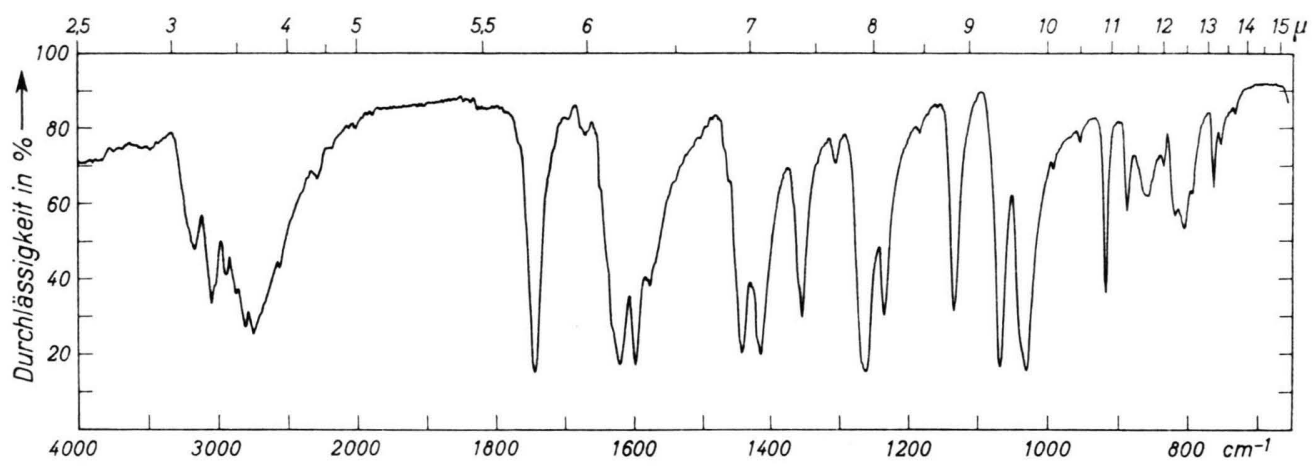

Abb. 8. IR-Spektrum des Benzochinon-4-oxim-3-carbonsäure-methylesters in $\mathrm{KBr}$.

\section{Benzochinon-4-oxim-3-carbonsäure}

$8 \mathrm{~g}$ 6-Nitro-3-oxybenzaldehyd werden in einem wasserfreien Gemisch von $180 \mathrm{ml}$ Benzol mit $40 \mathrm{ml}$ Aceton unter Erwärmen gelöst und in verschlossenem Glasgefäß etwa 2 Tage direkter Sonnenbestrahlung ausgesetzt; dann sind ca. 7,5 g Säure ausgefallen. Zur Säuberung genügt oft die Extraktion unveränderten Aldehyds mit warmem Aceton, sonst Umkristallisation aus Dioxan. Gelbe Kristalle, die ab $170^{\circ} \mathrm{C}$ unter Braunfärbung zusammensintern. Gefunden: C $50,7, \mathrm{H} 3,1 \%$; ber. für $\mathrm{C}_{7} \mathrm{H}_{5} \mathrm{NO}_{4}$ : C 50,3, H 3,0 Prozent.

\section{6-Nitro-3-oxybenzaldehyd-dimethylacetal}

10 g 6-Nitro-3-oxybenzaldehyd läßt man 24 Std. mit $70 \mathrm{ml}$ 1-proz. methanolischer Salzsäure im Dunkeln stehen; dann wird, mit viel Wasser versetzt, die organische Phase in Äther aufgenommen und der Äther nach Trocknen abgedampft. Gelbe Kristalle, Schmp. $77^{\circ} \mathrm{C}$, Kontrolle der Acetalbildung durch IR-Spektrum.

\section{Benzochinon-4-oxim-3-carbonsäuremethylester}

a) $4 \mathrm{~g}$ 6-Nitro-3-oxybenzaldehyd-dimethylacetal werden im wasserfreiem Gemisch von $80 \mathrm{ml}$ Benzol mit $0,5 \mathrm{ml}$ Methanol gelöst. Nach 4-stdg. Sonnenbestrahlung werden die ausgefallenen Kristalle abgetrennt (vornehmlich Oximcarbonsäure); bei weiterer Sonnenbestrahlung (insgesamt 2 Tage) fallen jeweils nach Ausfrieren insgesamt 2,5 g Substanz aus. Nach zweimaligem Umkristallisieren aus Dioxan/Benzol (1:1) braungelbe rhombische Kristalle, die zur Zwillingsbildung neigen. Schmp. 146 bis $150{ }^{\circ} \mathrm{C}$ (Zers.). Gefunden C $53,0, \mathrm{H} 4,0 \%$; berechnet für $\mathrm{C}_{8} \mathrm{H}_{7} \mathrm{NO}_{4}$ : C 53,0, $\mathrm{H} 3,9$ Prozent. b) 1,8 g Benzochinon-4-oxim-3-carbonsäure werden in $45 \mathrm{ml}$ wasserfreiem Methanol unter Erwärmen gelöst, und die Lösung dann auf $-18{ }^{\circ} \mathrm{C}$ gekühlt. Unter turbulentem Rühren trägt man nun ca. $50 \mathrm{ml} n / 2$-ätherischer Diazomethanlösung innerhalb 10 Min. ein. Die Lösungsmittel werden im Vakuum verdampft, der verbleibende Rückstand dreimal mit $50 \mathrm{ml}$ Petroläther extrahiert und dann zweimal aus Dioxan/Benzol 1:1 umkristallisiert: braungelbe Kristalle des Chinonoximcarbonsäuremethylesters. Der Petrolätherextrakt wird eingedampft und der Rückstand zweimal aus Petroläther umkristallisiert: gelbweiße faserige Kristalle, Schmp. $85^{\circ} \mathrm{C}$, des Benzochinon-4-oximmethyläther-3-carbonsäuremethylesters. Gefunden C 55,7, H 4,9\%; berechnet für $\mathrm{C}_{9} \mathrm{H}_{9} \mathrm{NO}_{4}$ : C 55,4, $\mathrm{H} 4,7$ Prozent.

Alle Protonenresonanz-Spektren wurden mit den Varian-NMR-Spektrographen A 60 und HA 100 aufgenommen. Verwendet wurden 3- bis 12-proz. Lösungen mit Tetramethylsilan als innerem Standard.

Die Aufnahme der UV-Spektren erfolgt mit dem Zeiss-Spektralphotometer mit Doppelmonochromator, die der IR-Spektren mit dem Leitz IR-Spektrographen.

Herrn Prof. Dr. W. Theilacker danke ich für sein Interesse an dieser Arbeit und für ideelle und materielle Unterstützung. Frau Dorle Götz und Frau MARgarethe Meyer haben viele Präparate angefertigt und die teilweise mühseligen Trennungen verschiedener Kristallformen durchgeführt. Herr HANS GüNTHER hat mit großer Geduld zahlreiche NMR-Spektren aufgenommen. Auch ihnen sei an dieser Stelle gedankt.

Der Deutschen Forschungsgemein $\mathrm{sch}$ a ft, Bad Godelberg, danke ich für die Überlassung des Varian A 60 Spektrometers, den F a r be n$\mathrm{f}$ a b rike n B a y e r, Leverkusen, für die Überlassung einiger Phenole. 